International Journal of Pure and Applied Mathematics

Volume 99 No. 3 2015, 325-341

ISSN: 1311-8080 (printed version); ISSN: 1314-3395 (on-line version)

url: http://www.ijpam.eu

doi: http://dx.doi.org/10.12732/ijpam.v99i3.8

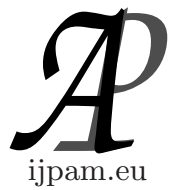

\title{
THE DENSITY OF THE PROCESS OF COLAESCING RANDOM WALKS
}

\author{
Samir Brahim Belhaouari \\ Department of Mathematics \\ Alfaisal University \\ P.O. Box 50927, Riyadh, 11533, SAUDI ARABIA
}

\begin{abstract}
We study the density of the process of coalescing random walks starting from $\mathbb{Z}$ at time 0 , where the random walk kernel associated to this model has finite second moment. It is shown that the density equals the survival probability of voter model with the initial condition being all 0's except for a single 1 at the origin and it converges to $\frac{1}{\sqrt{t}}$.
\end{abstract}

AMS Subject Classification: 60G05, 60G40, 60A10

Key Words: random walk, coalescing random walks, Voter model

\section{Introduction}

The paper is a continuity work of our previous papers realted to the tightness and to the evaluation of the interface for the one-dimensional voter model [11] and [12].

The voter model was introduced independently by Clifford and Sudbury (1973) and by Holley and Liggett (1975), and it has been used to model such diverse phenomena as the voting patterns in a population and the interaction between divergent genotypes (see Harisson 1990 [6]).

Received: November 10, 2014

(c) 2015 Academic Publications, Ltd. url: www.acadpubl.eu 
We consider the voter model $\left(\eta_{t}\right)_{t \geq 0}$ on a configuration space $\{0,1\}^{\mathbb{Z}}$. Each site $x \in \mathbb{Z}$ is occupied by an individual, and the values 0 or 1 denote for instance two different political opinions an individual can adopt.

The transition mechanism is specified by the function

$$
C(x, \eta)= \begin{cases}\sum_{y} q(x, y) \eta(y) & \text { if } \eta(x)=0, \\ \sum_{y} q(x, y)(1-\eta(y)) & \text { if } \eta(x)=1,\end{cases}
$$

where $q(x, y) \geq 0$ for $x, y \in \mathbb{Z}$ and

$$
\sum_{y} q(x, y)=1 \text { for } x \in \mathbb{Z} .
$$

The function $C$ represents the rate at which the coordinate $\eta(x)$ flips from 0 to 1 or from 1 to 0 when the system is in the state $\eta$.

The voter model is also formally described by the generator $\mathcal{A}$ acting on cylinder function $F:\{0,1\}^{\mathbb{Z}} \rightarrow \mathbb{R}$ (i.e., $F$ depends only on a finite number of coordinates of $\mathbb{Z}$ )

$$
(\mathcal{A} F)(\eta)=\sum_{x} \sum_{y} q(x, y) I_{\eta(x) \neq \eta(y)}\left(F\left(\eta^{x}\right)-F(\eta)\right), \quad \eta \in\{0,1\}^{\mathbb{Z}},
$$

where

$$
\eta^{x}(z)= \begin{cases}\eta(z) & \text { if } z \neq x \\ 1-\eta(z) & \text { if } z=x\end{cases}
$$

The process $\eta_{t}$ satisfies as

$$
P^{\eta}\left(\eta_{t}(x) \neq \eta(x)\right)=C(x, \eta) t+o(t)
$$

as $t \downarrow 0$, for all $x \in \mathbb{Z}$, and for all $\eta \in\{0,1\}^{\mathbb{Z}}$.

Furthermore we require that in each transition only one coordinate changes after an infinitesimal interval of time, i.e.,

$$
P^{\eta}\left(\eta_{t}(x) \neq \eta(x), \eta_{t}(y) \neq \eta(y)\right)=o(t)
$$

as $t \downarrow 0$, for all each $x, y \in \mathbb{Z}$, with $x \neq y$ and for all $\eta \in\{0,1\}^{\mathbb{Z}}$.

An equivalent way to describe the rate of the voter model is to say that site $x$ waits an exponential time with parameter one, after that it flips to the value it sees at that time at a site $y$ which is chosen with probability $q(x, y)$.

Let us consider the translation invariant setting, that is, $q(x, y)=p(y-x)$. We define $p(x)=q(0, x)$. We also suppose that $p(x)=p(-x)$, for all $x \in$ $\mathbb{Z}$, because, as we will see, we are interested in our study of tightness in the 
difference of two independent random walks, which necessarily is a symmetric random walk.

It is clear that point masses on the configuration $\eta \equiv 1$ and $\eta \equiv 0$ are invariant for the process. Thus the voter model is never ergodic (we say the process is ergodic if there only one invariant measure ).

For $0<\theta<1$ let the initial distribution $\mathcal{L}\left[\eta_{0}\right]=v$ be a translation invariant, spatially ergodic measure $v$ with intensity

$$
v\left\{\eta \in\{0,1\}^{\mathbb{Z}}: \eta(x)=1\right\}=\theta, \quad \text { for all } x \in \mathbb{Z} .
$$

The following basic result can be found in [8].

Basic ergodic theorem:

(i) If $p$ is recurrent:

$$
\mathcal{L}^{v}\left[\eta_{t}\right] \Rightarrow_{t \rightarrow \infty} \theta \delta_{\underline{1}}+(1-\theta) \delta_{\underline{0}},
$$

where $\underline{0}$ or $\underline{1}$ denotes the configuration with all sites 0 or all sites 1 .

(ii) If $p$ is transient, there exists a unique probability measure $\mu_{\theta}$, depending only on $\theta$, such that

$$
\mathcal{L}^{v}\left[\eta_{t}\right] \Rightarrow_{t \rightarrow \infty} \mu_{\theta} .
$$

This measure $\mu_{\theta}$ has the following properties:

a) $\mu_{\theta}$ is an invariant measure of the process $\left(\eta_{t}\right)_{t \geq 0}$,

b) $\mu_{\theta}$ is ergodic,

c) let $\eta_{\infty}$ have the distribution $\mu_{\theta}$, then for all $x, y \in \mathbb{Z}$ :

$$
\begin{gathered}
E\left(\eta_{\infty}(x)\right)=\theta \\
E\left(\eta_{\infty}(x) \eta_{\infty}(y)\right) \rightarrow \theta^{2} \text { as }\|x-y\| \rightarrow \infty,
\end{gathered}
$$

where the symbol $\Rightarrow$ denotes weak convergence.

Thus the process has only the two trivial extremal invariant measures if $p$ is recurrent, but has a full one-parameter family of them if $p$ is transient. For the more general detail see [7] and [2].

For technical reasons in the study of interacting particle systems, we introduce the notion of the duality. Typically, the duality relation associates a dual process with the process of interest in such a way that problems involving the original process can be reformulated as problems involving the dual process. If the dual process is substantially simpler than the original process or if the 
nature of the problems changes in useful way, then the reformulated problems may be easier to deal with than the original problems.

The general definition of duality is if we have two Markov process with state spaces, $X$ for the process $\eta_{t}$ and $Y$ for the process $\zeta_{t}$, and let $H(\eta, \zeta)$ be a bounded measurable function on $X \times Y$. The process $\eta_{t}$ and $\zeta_{t}$ are said to be dual one another with respect to $H$ if

$$
E^{\eta} H\left(\eta_{t}, \zeta\right)=E^{\zeta} H\left(\eta, \zeta_{t}\right)
$$

The coalescing random walks and the voter model are dual with respect to $H$, fore more detail see [5] and [8].

System of coalescing random walks, which arise here as dual of the voter model, have been studied in their own right. Papers on this subject are Erdos and Ney (1974), Schawartz (1976), Griffeath (1978), Bramson and Griffeath (1980), and Arratia $(1981,83)$.

We denote by $\xi_{t}^{A}$ the process of continuous time coalescing random walks on $\mathbb{Z}$ associated to the transition probability $p($.$) , which consists of a collections$ of random walks starting from each site $x \in A \subseteq \mathbb{Z}$.

These particles undergo independent continuous time rate one random walks with $p: \mathbb{Z}^{2} \rightarrow[0,1]$ as a transition function, except that whenever a particle jumps to a site which is already occupied by another, the two particles coalesce into one.

The coalescing model is formally described by the generator $\mathcal{A}$ acting on cylinder function $F$ (i.e. $F$ depends on only a finite number of coordinates of $\mathbb{Z})$,

$$
(\mathcal{A} F)(\xi)=\sum_{x} \sum_{y} p(x, y) \xi(x)\left(F\left(\xi^{x}\right)-F(\xi)\right), \quad \xi \in\{0,1\}^{\mathbb{Z}},
$$

where

$$
\xi^{x}(z)= \begin{cases}1 & \text { if } z=y \\ 0 & \text { if } z=x \\ \xi(z) & \text { otherwise }\end{cases}
$$

as rate of the coalescing random walks, $\xi(x)=1$ it means that there is a particle at the site $x$ at this time.

The duality relation between the two process asserts that

$$
\left\{\eta_{t}(x)=1, \forall x \in A\right\}=\left\{\eta(x)=1, \forall x \in \xi_{t}^{A}\right\}
$$

(see $[3]$ and $[8])$. 
To better understand this result we use the graphical representation which provide an important link between coupling and coalescing duality for spin systems which have a certain additive properties i.e.,

$$
\eta_{t}^{\eta \cup \xi}=\eta_{t}^{\eta} \cup \eta_{t}^{\xi}, \text { for all } \eta, \xi \in\{0,1\}^{\mathbb{Z}},
$$

where $\eta \cup \xi=\eta \vee \xi$ i.e., $\eta(x) \cup \xi(x)=\eta(x) \vee \xi(x)$.

The coupling is simply a construction of two or more stochastic processes on a commun probability space, of course, the coupling will normally only be useful if the mechanisms of the various stochastic processes are connected in a nontrivial way.

To construct the voter model, let $\left(T_{n}^{x}\right)_{n \geq 1}$, for all $x \in \mathbb{Z}$ be independent poisson process with rate 1 , and let $\left(Y_{n}^{x}\right)_{n \geq 1}$ be i.i.d. sequence with $P\left(Y_{n}^{x}=y\right)=p(x, y)$ for all $y \in \mathbb{Z}$. In making these definitions, we have in mind that at time $T_{n}^{x}$ the voter at $x$ decides for the nth time to change her mind, and she adopts at time $T_{n}^{x}$ the opinion of the voter at $Y_{n}^{x}$. For future developments on mind, we will use a special recipe. For each $x$ and $n$, we draw an arrow from $\left(T_{n}^{x}, Y_{n}^{x}\right)$ to $\left(x, T_{N}^{x}\right)$, and write a $\delta$ at $\left(x, T_{n}^{x}\right)$ (see [3])

To construct the process from this "graphical representations", we imagine fluid entering the bottom at the point in $\eta_{0}$ and the following up the structure. The $\delta$ 's are dams and the arrows are pipes lines which allow the fluid to flow in the indicated direction.

We say that is a path from $(x, 0)$ to $(y, t)$ if there is a sequence of time $S_{0}=$ $0<S_{1}<S_{2} \ldots S_{n}<S_{n+1}=t$ and special locations $x_{0}=x, x_{1}, \ldots, x_{n}, x_{n+1}=y$ so that:

i) for $i=1, \ldots, n$ there is an arrow from $x_{i-1}$ to $x_{i}$ at time $S_{i}$ and

ii) the vertical $\left\{x_{i}\right\} \times\left(S_{i}, S_{i+1}\right), i=0,1, \ldots, n$ don't contains any $\delta$ 's.

Since every individual at time $t$ has adopted the opinion of some individual at time 0 , it follows that

$$
\eta_{t}^{A}=\{y: \text { for some } x \in A \text { there is a path form }(x, 0) \text { to }(y, t)\},
$$

which is the set of sites that have opinion 1 if the initial configuration is $\eta_{0}^{A}$ defined by $\eta_{0}^{A}(x)=1$ if $x \in A$.

To Construct the dual process, reverse that direction of the arrows in the graphical presentation and change time by mapping $\widehat{s}=t-s$. If we define 
paths as before, and let

$$
\widehat{\xi}_{t}^{B}=\{x: \text { for some } y \in B \text { there is a path from }(y, \widehat{0}) \text { to }(x, \widehat{t})\} .
$$

Then it is clear that

$$
\left\{\widehat{\xi}_{t}^{B} \cap A \neq \emptyset\right\}=\left\{\eta_{t}^{A} \cap B \neq \emptyset\right\} .
$$

We define a process $\widetilde{\xi}_{t}$ that has the same distribution as $\widehat{\xi}_{t}$, and let

$$
\widetilde{\xi}_{t}^{B}=\{x: \text { for some } y \in B \text { there is a path from }(y, 0) \text { to }(x, t)\} .
$$

So we can write that

$$
P\left(\eta_{t}^{A} \cap B \neq \emptyset\right)=P\left(\widetilde{\xi}_{t}^{B} \cap A \neq \emptyset\right)
$$

If we think of 1 's as particles and 0 's as empty sites, then $\widetilde{\xi}_{t}$ may be described as "Coalescing random walks". A $\delta$-arrows from $x$ to $x+y$ causes a particle at $x$ to jump to $x+y$. If the site $x+y$ is occupied, the two particles coalesce to 1 , which is the property of Coalescing random walks.

Using the duality between the voter model and the coalescing random walks, we can write from the equation (1) for $A=\mathbb{Z}$ and $B=\{0\}$

$$
P\left(0 \in \xi_{t}^{\mathbb{Z}}\right)=P\left(\eta_{t}^{0} \neq \emptyset\right), \quad t \geq 0,
$$

which it mean that the particle density of $\xi_{t}^{\mathbb{Z}}$ equals the survival probability of $\eta_{t}^{0}$.

Using the range of the random walk we show the following Theorem

Theorem 1.1. Let $\xi_{t}^{\mathbb{Z}}$ be the process of coalescing random walks starting from $\mathbb{Z}$ at time 0 , where the random walk kernel associated to this model has finite second moment. Let $\eta_{t}^{0}$ be the voter model dual to $\xi_{t}^{\mathbb{Z}}$ with the initial condition being all 0 's except for a single 1 at the origin. Then

$$
P\left(0 \in \xi_{t}^{\mathbb{Z}}\right)=P\left(\eta_{t}^{0} \neq \emptyset\right) \sim \frac{1}{\sigma \sqrt{t}}
$$

We denote the property $\exists c_{1}>0, c_{2}>0$ and $n_{0}>0$ such that for every $n>n_{0}: c_{1} B_{n} \leq A_{n} \leq c_{2} B_{n}$, by a simple notation $A_{n} \sim B_{n}$, we denote positive constants which do not depend on $n$ by $c_{i}$. Given two collections of sequences indexed by $k, A_{n}^{k}, B_{n}^{k} n=1,2,3, \ldots$ we say that $A_{n}^{k} \sim B_{n}^{k}$ uniformly in $k$ if for each fixed $k, A_{n}^{k} \sim B_{n}^{k}$ and the relevant $n_{0}, c_{1}, c_{2}$ can be chosen independent of $k$. 


\section{Preliminary Results}

In this section we will give some background on random walks which are necessary to study and understand the voter model, after that we will state some well known properties of random walks, most of which come from the local central limit theorem for sums of i.i.d. random variables with second moment. The standard reference for this chapter is [10].

After that we will prove the two following lemmas which are useful in our study.

Lemma 2.1. Let $\left(X_{n}\right)_{n \geq 0}$ be a discrete time, symmetric, random walk with transition function $p(x)$, with finite second moment, then

a. $\forall x \neq 0: P^{0}\left(\tau_{x}<\tau_{0}\right) \sim 1 /|x|$.

b. $P^{0}\left(\tau_{0} \geq n\right) \sim 1 / \sqrt{n}$.

c. Uniformly in $x \neq 0, P^{x}\left(\tau_{0} \geq n\right) \sim(|x| / \sqrt{n}) \wedge 1$.

In the case of continuous processes, the results remain the same, with $n \in \mathbb{N}$ replaced by a continuous time parameter $t \geq 0$.

We define the range of the random walk at time $t$ as the number of the distinct sites visited at least once by the random walk before time $t$.

In order to prove the theorem 1.1, we need to show the following lemma.

Lemma 2.2. Let $R_{t}$ be the range of a continuous random walk with transition function $p(x)$, if $\sum_{x}|x|^{2} p(x)<\infty$ then

$$
E\left(R_{t}\right) \sim \begin{cases}\sqrt{t} & \text { if } d=1 \\ \frac{t}{\log (t)} & \text { if } d=2 \\ \gamma_{d} t & \text { if } d>2\end{cases}
$$

where $\gamma_{d}=P^{0}\left(\tau_{0}>t\right)$.

This lemma extends Theorem 1' of M. Bramsom and D. Griffeath (1980) [1], which they applied a certain result of Sawyer's (1979) to show this result for $d \geq 2$. As we will see in the proof of this lemma that $E\left(R_{t}\right)=1+\int_{0}^{t} P^{0}\left(\tau_{0}>\right.$ $s) d s$, then to prove this lemma it suffice to extend the Lemma 2.1.b for $d>1$.

\subsection{Background on Random Walk on $\mathbb{Z}^{d}$}

Let $\left(X_{n}\right)_{n \geq 1}$ be a sequence of $\mathbb{Z}^{d}$-valued independent i.i.d. random variables, defined on a probability space $(\Omega, \mathcal{F}, P)$. The set $\Omega$ could be taken as a set of infinite sequences $w=\left(w_{1}, w_{2}, \ldots\right)$, with each $w_{i} \in \mathbb{Z}^{d}$. 
The $\sigma$-field $\mathcal{F}$ is the smallest $\sigma$-field which contains every cylinder set, i.e., set $A_{n}$ of the form $A_{n}=\left\{w: w_{k}=a_{k}, k=1,2, \ldots, n\right\}, n \geq 0, a_{k} \in \mathbb{Z}^{d}$. The sequence $\left(S_{n}\right)_{n \geq 0}$ defined by $S_{0}=0, S_{n}=\sum_{i=1}^{n} X_{i}$ is called a homogeneous translation invariant random walk, thus $S_{n}(\omega)=\sum_{i=1}^{n} \omega_{i}$.

We define the probability measure $P$ on $(\Omega, \mathcal{F})$ by

$$
P\left(A_{n}\right)=q\left(0, a_{1}\right) q\left(0, a_{2}\right) \ldots q\left(0, a_{n}\right), n \geq 0
$$

where $q(x, y)$ is the transition function of random walk which has the property that $q(x, y)=q(y-x) \geq 0$ for any $x, y \in \mathbb{Z}^{d}$, and $\sum_{x} q(y, x)=1$ for all $y \in \mathbb{Z}^{d}$.

We denote by $q_{n}(0, x)$ the probability of an "n-step" transition from 0 to $x$, i.e., $q_{n}(0, x)=P\left(S_{n}=x\right)$.

\subsubsection{Recurrence}

We will investigate the question "Does the sequence $S_{1}, S_{2}, \ldots$ return to 0 infinitely often?" The answer of this question is either yes or no, and the random walk is called recurrent or transition accordingly.

The recurrence of a random walk manifest itself in the limiting behavior of $G_{n}(0,0)=\sum_{k=1}^{n} q_{k}(0,0)$, but it is not clear exactly how $G_{n}(0, x)$ depends on the recurrence of the random walk. Recall that $G(0,0)=\lim _{n \rightarrow \infty} G_{n}(0,0)$ is called the green's function of the random walk.

Since $G(0, x) \leq G(0,0) \leq \infty$ for every $x$ (see P.1.3 [10]), it is clear that $G(0, x)<\infty$ for all $x$ if the random is transient, but suppose that random walk is recurrent, so that $G(0,0)=\infty$, does it follow that $G(0, x)=\infty$ for every $x$ ? The answer depends on whether that the random is irreducible or not. We define three subsets of $\mathbb{Z}^{d}$ called $\Sigma, \Sigma^{+}$and $\bar{\Sigma}$

$$
\begin{aligned}
\Sigma & =\{x: q(0, x)>0\} \\
\Sigma^{\prime} & =\left\{x: q_{n}(0, x)>0 \text { for some } n \geq 0\right\} \\
\bar{\Sigma} & =\left\{x: x=y-z \text { for some } y, z \in \Sigma^{\prime}\right\} .
\end{aligned}
$$

A random walk is irreducible if $\bar{\Sigma}=\mathbb{Z}^{d}$. Finally we summarize the result as the following theorem

Theorem 2.3. Let $q(x, y)$ be the transition function of irreducible random walk. Then there are only two possibilities:

(i) Transient case

$$
\forall x \in \mathbb{Z}^{d}: G(0, x)<\infty
$$


(ii) Recurrent case

$$
\forall x \in \mathbb{Z}^{d}: G(0, x)=\infty .
$$

Remark 2.4. If $x \in \mathbb{Z}^{d}-\Sigma^{\prime}$ this implies that $G(0, x)=0$.

Now we turn instead to the task of finding a sufficient condition for recurrence. Restricting attention to one-dimensional random walk. Let $m=$ $\sum_{x}|x| q(0, x)$ and in case $m<\infty$ let $\mu=\sum_{x} x q(0, x)$

Theorem 2.5. One-dimensional translation invariant random walk with finite absolute mean $m$ is recurrent if and only if the mean $\mu=0$.

For the proof see Chung-Fuchs theorem [4] and [10].

\subsubsection{Harmonic Analysis}

Given a random walk which is completely specified by its transition function $q(x, y)$ on $\mathbb{Z}^{d}$, we make definition that the characteristic function of the random walk is

$$
\phi(\theta)=E\left(e^{i X . \theta}\right)=\sum_{x} q(0, x) e^{i x \cdot \theta}, \theta \in \mathbb{R} .
$$

By independence we have

$$
\begin{aligned}
\phi_{n}(\theta) & =E\left(e^{i S_{n} \cdot \theta}\right) \\
& =\phi^{n}(\theta) \\
& =\sum_{x} q_{n}(0, x) e^{i x \cdot \theta}, \theta \in \mathbb{R}^{d}, n \geq 1,
\end{aligned}
$$

from where $q_{n}(0, x)$ can be implicitly obtained as the inverse Fourier transformation of $\phi^{n}(\theta)$ :

$$
q_{n}(0, x)=\left(\frac{1}{2 \pi}\right)^{d} \int_{\mathbb{R}} e^{-i x \cdot \theta} \phi^{n}(\theta) d \theta, \forall x \in \mathbb{Z}^{d} .
$$

In [10], it was established a simple criterion for aperiodicity in terms of the characteristic function:

i) The random walk on $\mathbb{Z}^{d}$, is irreducible if and only if its characteristic function $\phi(\theta)$, defined for $\theta$ in $\mathbb{R}^{d}$, has the following property

$$
\phi(\theta)=1 \Leftrightarrow \theta=\left(2 \pi k_{1}, \ldots, 2 \pi k_{d}\right), \quad k_{i} \in \mathbb{Z}^{d} \text { for } i=1, \ldots, d .
$$


ii) For aperiodic random walk, there exists a constant $C>0$ such that

$$
1-\operatorname{Re} \phi(\theta) \geq C|\theta|^{2}, \text { for all }|\theta| \leq \pi
$$

Using the characteristic function, we can state the asymptotic behavior of the transition function $q_{n}(0, x), x \in \mathbb{Z}^{d}$.

Since the dimension is the crucial thing, it will be useful to introduce a new class of random walk. We say a random walk is genuinely $d$-dimensional if the group $\bar{\Sigma}$ associated with the random walk is $d$-dimensional. For every genuinely d-dimensional random walk with the single exception in one-dimensional of these where $q(0, x)=1$ for some $x \in \mathbb{Z}^{d}$, satisfies

$$
q_{n}(0, x) \leq \frac{c}{\sqrt{n}}, \quad x \in \mathbb{Z}^{d}, n \geq 1
$$

for some $c>0$.

\subsection{Proof of Lemma 2.1}

a. Let $g_{0}$ be the green function for the process killed at 0 , defined as follows:

$$
g_{0}(x, y)= \begin{cases}\sum_{n=0}^{\infty} P^{x}\left(X_{n}=y ; \tau_{0}>n\right) & \text { if } x \text { and } y \in \mathbb{Z}-\{0\} \\ 0 & \text { otherwise }\end{cases}
$$

Let, for $m \geq 1, \tau_{x}^{m}=\inf \left\{r>\tau_{x}^{m-1}\right.$ so that $\left.X_{r}=x\right\}$, with $\tau_{x}^{0}=0$ and $\tau_{x}^{1}=\tau_{x}$.

Then for $x \neq 0$ :

$$
\begin{aligned}
g_{0}(x, x) & =\sum_{n=0}^{\infty} P^{x}\left(X_{n}=x ; \tau_{0}>n\right) \\
& =1+\sum_{n=1}^{\infty} P^{x}\left(X_{n}=x ; \tau_{0}>n\right) \\
& =1+\sum_{n=1}^{\infty} \sum_{m=1}^{n} P^{x}\left(\tau_{x}^{m}=n ; \tau_{0}>n\right) \\
& =1+\sum_{m=1}^{\infty} P^{x}\left(\tau_{x}^{m}<\tau_{0}\right),
\end{aligned}
$$

by the Markov property we have

$$
g_{0}(x, x)=1+\sum_{m=1}^{\infty}\left(P^{x}\left(\tau_{x}<\tau_{0}\right)\right)^{m}
$$




$$
=\frac{1}{P^{x}\left(\tau_{0}<\tau_{x}\right)} .
$$

We now observe that $g_{0}(x, x)=a(x)+a(-x)-a(0)$, (for more detail see Lemma ?? of the Appendix and P29.4 [10]), where

$$
a(x)=\sum_{i=0}^{\infty}\left(P^{0}\left(X_{i}=0\right)-P^{x}\left(X_{i}=0\right)\right)
$$

is the potential kernel function.

Since by symmetry $P^{x}\left(\tau_{0}<\tau_{x}\right)=P^{0}\left(\tau_{x}<\tau_{0}\right)$, to finish the proof, it suffices to show that $P^{x}\left(\tau_{0}<\tau_{x}\right)=\frac{1}{2 a(x)} \sim \frac{1}{|x|}$.

Let $a_{n}(x)=\sum_{i=0}^{n}\left(P^{0}\left(X_{i}=0\right)-P^{x}\left(X_{i}=0\right)\right)$, so that $a(x)=\lim _{n \rightarrow \infty} a_{n}(x)$.

Let $\phi(\theta)$ the characteristic function of the random walk, then

$$
\begin{aligned}
a_{n}(x)+a_{n}(-x) & =\sum_{k=0}^{n} \frac{1}{\pi} \int_{-\pi}^{\pi}[1-\cos (x \theta)] \phi^{k}(\theta) d \theta, \quad \text { (see P6.3 [10]) } \\
& =\frac{1}{\pi} \int_{-\pi}^{\pi} \frac{1-\cos (x \theta)}{1-\phi(\theta)}\left[1-\phi^{n+1}(\theta)\right] d \theta \\
\Rightarrow 2 a(x) & =\lim _{n \rightarrow \infty} \frac{1}{\pi} \int_{-\pi}^{\pi} \frac{1-\cos (x \theta)}{1-\phi(\theta)}\left[1-\phi^{n+1}(\theta)\right] d \theta \\
& =\frac{1}{\pi} \int_{-\pi}^{\pi} \frac{1-\cos (x \theta)}{1-\phi(\theta)} d \theta<\infty,(\text { because the integral converges) } \\
& \sim \frac{1}{\pi} \int_{-\pi}^{\pi} \frac{2 \sin ^{2}(x \theta / 2)}{\theta^{2}} d \theta, \quad(\text { see P6.7 and P7.5, [10]) } \\
& \sim \frac{|x|}{\pi} \int_{-x \pi / 2}^{x \pi / 2} \frac{\sin ^{2} y}{y^{2}} d y .
\end{aligned}
$$

For $|x| \geq 1$, we have $\int_{-\pi / 2}^{\pi / 2} \frac{\sin ^{2} y}{y^{2}} d y \leq \int_{-x \pi / 2}^{x \pi / 2} \frac{\sin ^{2} y}{y^{2}} d y \leq \int_{-\infty}^{\infty} \frac{\sin ^{2} y}{y^{2}} d y=\pi$.

Thus

$$
a(x) \sim|x|
$$

and we have the required result (for more detail see proofs of P28.4 and of P28.8, [10]).

b. The result follows from P.32.3, [10],

$$
\lim _{n \rightarrow \infty} \sqrt{n} P^{0}\left(\tau_{0}>n\right)=\sqrt{\frac{2}{\pi}} \sigma .
$$


c. We deduce from $\mathbf{b}$ that $\exists c_{1}>0$ and $c_{2}>0$, such that for each positive integer $n, c_{2} / n \leq P^{0}\left(\tau_{0} \geq n^{2}\right) \leq c_{1} / n$.

We observe also that

$$
\begin{aligned}
& P^{0}\left(\tau_{0}>n^{2}\right) \geq P^{0}\left(\tau_{x}<\tau_{0}, \tau_{0}>n^{2}\right) \\
& P^{0}\left(\tau_{0}>n^{2}\right) \geq P^{0}\left(\tau_{x}<\tau_{0}\right) P^{x}\left(\tau_{0}>n^{2}\right) .
\end{aligned}
$$

Therfore

$$
\begin{aligned}
P^{x}\left(\tau_{0}>n^{2}\right) & \leq \frac{P^{0}\left(\tau_{0}>n^{2}\right)}{P^{0}\left(\tau_{x}<\tau_{0}\right)} \\
& \leq \frac{c_{1}}{n P^{0}\left(\tau_{x}<\tau_{0}\right)} \\
& \leq c_{3} \frac{|x|}{n}
\end{aligned}
$$

where the inequality follows from $b$. and a respectively.

So $P^{x}\left(\tau_{0}>n^{2}\right) \leq c_{3}\left(\frac{|x|}{n} \wedge 1\right)$ for $c_{3}$ not depending on $x$ or $n$.

In the other way we use a last time at 0 decomposition, we have

$$
\begin{aligned}
& P^{x}\left(\tau_{0} \leq n^{2}\right)=\sum_{k=0}^{n^{2}} P_{k}(x, 0) P^{0}\left(\tau_{0}>n^{2}-k\right) \quad \text { for } x \neq 0 \\
& P^{0}\left(\tau_{0} \leq n^{2}\right)=\sum_{k=0}^{n^{2}} P_{k}(0,0) P^{0}\left(\tau_{0}>n^{2}-k\right)=1 .
\end{aligned}
$$

Thus

$$
\begin{aligned}
P^{x}\left(\tau_{0}>n^{2}\right) & =\sum_{k=0}^{n^{2}}\left(P_{k}(0,0)-P_{k}(x, 0)\right) P^{0}\left(\tau_{0}>n^{2}-k\right) \\
& \geq P^{0}\left(\tau_{0}>n^{2}\right) a_{n^{2}}(x) \\
& \geq \frac{c|x|}{n} \wedge 1 .
\end{aligned}
$$

The extension of these results to continuous time processes is either automatic, as part a, or trivial $\left(a(x)=\int_{0}^{\infty}\left(P^{0}\left(x_{t}=0\right)-P^{0}\left(x_{t}=x\right)\right) d t\right.$, and also $\left.g_{0}(x, x)=\int_{0}^{\infty} P^{x}\left(x_{t}=x ; \tau_{0}>t\right) d t\right)$.

In order to prove Theorem 1.1, we begin by proving the Lemma 2.2. 


\subsection{Proof of Lemma 2.2}

Let $\gamma_{d}(t)$ be the probability that at time $t$, the process $X(t)$ takes a position it has not visited before by the time of the last jump before time $t$, then

$$
\gamma_{d}(t)=\sum_{f=1}^{\infty} P\left(X\left(\sigma_{i}\right) \neq X\left(\sigma_{f}\right) \quad \text { for } i<f ; \sigma_{f} \leq t<\sigma_{f+1}\right)
$$

where $\sigma_{i}$ is the time of the $\mathrm{i}$-th jump of $\mathrm{X}(\mathrm{t})$. Due to symmetry and independence we have:

$$
\begin{aligned}
\gamma_{d}(t) & =\sum_{f \geq 1} P^{0}\left(X\left(\sigma_{f-i}\right) \neq 0 \text { for } 0<i<f ; \sigma_{f} \leq t<\sigma_{f+1}\right) \\
& =\sum_{f \geq 1} P^{0}\left(X\left(\sigma_{j}\right) \neq 0 \text { for } 0<j<f ; \sigma_{f} \leq t<\sigma_{f+1}\right) \\
& =P^{0}\left(X(s) \neq 0, \quad \forall \sigma_{1}<s \leq t\right) \\
& =P^{0}\left(\tau_{0}>t\right) .
\end{aligned}
$$

We remark that $\gamma_{d}(t)$ decreases as a function of $\mathrm{t}$.

Let $U_{d}(t)=P(X(t)=0)$, and by the local central limit theorem (see [4]), we can write that

$$
U_{d}(t) \sim\left(\frac{d}{2 t \pi}\right)^{d / 2} .
$$

Classifying the paths according to its last return to the origin, we have

$$
\begin{aligned}
1 & =\int_{0}^{t} P^{0}\left(X(s)=0 ; X\left(s^{\prime}\right) \neq 0 \forall s<s^{\prime} \leq t\right) d s \\
& =\int_{0}^{t} U_{d}(s) \gamma_{d}(t-s) d s .
\end{aligned}
$$

Case $d \geq 3$ we have

$$
U_{d}=\int_{0}^{\infty} U_{d}(s) d s<\infty
$$

as $\gamma_{d}(t)$ is a bounded decreasing function, which mean the limit exist, then

$$
\gamma_{d}=\lim _{t \rightarrow \infty} \gamma_{d}(t) .
$$

We have for $0<k \leq t$

$$
\gamma_{d}(t-k) \int_{0}^{k} U_{d}(s) d s+\int_{k}^{t} U_{d}(t) d t \geq 1
$$


Letting $k \rightarrow \infty$ so that $t-k \rightarrow \infty$ we got $\gamma_{d}(t-k) U_{d} \geq 1+o(1)$ which gives

$$
\gamma_{d} \geq \frac{1}{U_{d}}
$$

In other way we can write that $\gamma_{d}(t) \int_{0}^{t} U_{d}(s) d s \leq 1$, as $t \rightarrow \infty$ we have

$$
\gamma_{d} \leq \frac{1}{U_{d}}
$$

We conclude that

$$
\gamma_{d} \sim \frac{1}{U_{d}}
$$

Case $d=2$ we have

$$
U_{2}=\int_{0}^{t} U_{2}(s) d s \sim \log (t)
$$

We have as $t \rightarrow \infty$

$$
\gamma_{2}(t) \log (t) \leq 1+o(1)
$$

Similarly, for $0<k \leq t$, we have $\gamma_{2}(t-k) \int_{0}^{k} U_{2}(s) d s+\int_{k}^{t} U_{2}(s) d s \geq 1$.

Thus, if $k \rightarrow \infty$ and $t \rightarrow \infty$

$$
\gamma(t-k) \frac{\log (k)}{\pi}+\frac{\log (t / k)}{\pi} \geq 1+o(1)
$$

taking $k=t / 2-t /(\log (t / 2))$, we find

$$
\gamma_{2}(t) \geq \frac{(1+o(1)) \pi}{\log (t)} .
$$

We find that

$$
\gamma_{2} \sim \frac{\pi}{\log (t)}
$$

Case $d=1$ As before we can find that

$$
\gamma_{1} \sim \sqrt{\frac{1}{\pi t}}
$$

The expected number of different points covered during time $t$ is given by

$$
E\left(R_{t}\right)=1+\int_{0}^{t} \gamma_{d}(s) d s .
$$


Therefore we find the result

$$
\begin{aligned}
E\left(R_{t}\right) & \sim \sqrt{t}, \text { if } d=1 \\
& \sim \frac{\pi t}{\log (t)}, \quad \text { if } d=2 \\
& \sim \gamma_{d} t, \text { if } d \geq 3
\end{aligned}
$$

Remark: $\int \frac{d x}{\ln x} \sim \int^{x} \frac{\ln x-1}{\ln ^{2} x} d x \sim \frac{x}{\ln x}$ as $x \rightarrow \infty$.

\section{Proof of Theorem 1.1}

Let $n_{t}=\left|\eta_{t}^{0}\right|=\left|\left\{x: \eta_{t}^{0}(x)=\eta_{0}^{0}(0)=1\right\}\right|$ be the number of sites that have opinion 1 at time $t$.

So we can write $n_{t}=\sum_{x} I_{\eta_{t}^{0}(x)=1}$, then

$$
P\left(\eta_{t}^{0} \neq \emptyset\right)=P\left(n_{t}>0\right)
$$

By using the Cauchy Schwartz's inequality we have

$$
\begin{aligned}
n_{t} & =n_{t} I_{n_{t}>0} \\
E^{2}\left(n_{t}\right) & \leq E\left(n_{t}^{2}\right) P\left(n_{t}>0\right) \\
\Rightarrow P\left(n_{t}>0\right) & \geq \frac{E^{2}\left(n_{t}\right)}{E\left(n_{t}^{2}\right)}
\end{aligned}
$$

We note that

$$
E\left(n_{t}\right)=\sum_{x} P\left(\eta_{t}^{0}(x)=1\right)=\sum_{x} P\left(X_{t}^{x, t}=0\right)=1
$$

where $X_{t}^{x, t}$ is the dual process (we can prove easily that $n_{t}$ is a martingale).

$$
\begin{aligned}
E\left(n_{t}^{2}\right) & =\sum_{x, y} P\left(\eta_{t}^{0}(x)=\eta_{t}^{0}(y)=1\right) \\
& =\sum_{x, y} P\left(\xi_{t}^{x, y}=\{0\}\right) \\
& =\sum_{x, y} P\left(X_{t}^{x, t}=0, \tau^{X^{x}-X^{y}}<t\right) \\
& =\sum_{l_{1}, l_{2}} P\left(X_{t}^{0, t}=-l_{2}, \tau_{-l_{1}}^{X^{x}-X^{y}}<t\right)
\end{aligned}
$$




$$
\begin{aligned}
& =\sum_{l_{1}} P\left(\tau_{-l_{1}}^{X^{x}-X^{y}}<t\right) \\
& =E\left(R_{t}^{X^{x}-X^{y}}\right)=E\left(R_{2 t}^{X^{0}}\right) \\
& \sim \sqrt{t}(\text { see Lemma } 2.2) .
\end{aligned}
$$

Thus, there exists a constant $c>0$

$$
P\left(n_{t}>0\right) \geq \frac{c}{\sqrt{t}} .
$$

For the other direction, we remark that if $\sigma(t)=\inf \left\{s>t: \eta_{s} \neq \eta_{t}\right\}$, then for all $t \geq 0$

$$
P\left(n_{\sigma(t)}=n_{t}+1\right)=P\left(n_{\sigma(t)}=n_{t}-1\right)=\frac{1}{2},
$$

it is easy to see that

$$
P\left(n_{t+s}=k+1 \mid n_{t}=k\right)=P\left(n_{t+s}=k-1 \mid n_{t}=k\right) \text { as } s \downarrow 0,
$$

for $k \in \mathbb{N}^{*}$ Therefore we can say that $n_{t}$ is a non-homogeneous random walk, i.e. with variable rate $\lambda(t)$ which is bigger that 1, because the position of 1's in $\eta_{t}^{0}$ do not necessarily form only one block. Let $n_{t}^{\prime}$ be a simple random walk then

$$
\begin{aligned}
P\left(n_{t}>0\right) & =P\left(n_{t}>0, N_{t}<t / 2\right)+P\left(n_{t}>0, N_{t} \geq t / 2\right) \\
& =P\left(N_{t}<t / 2\right)+P\left(n_{t}>0, N_{t} \geq t / 2\right) \\
& \leq P\left(N_{t}<t / 2\right)+P\left(\tau_{0}^{n^{\prime}}>\lfloor t / 2\rfloor\right) \quad(\text { see Lemma 2.1) } \\
& \leq \epsilon_{t}+\frac{c^{\prime}}{\sqrt{t}} .
\end{aligned}
$$

where $\epsilon_{t}$ goes exponentially to zero as t goes to infinity. Then we conclude that

$$
P\left(n_{t}>0\right) \sim \frac{1}{\sqrt{t}} .
$$

Thus

$$
P\left(0 \in \xi_{t}^{\mathbb{Z}}\right) \sim \frac{1}{\sqrt{t}}
$$

\section{References}

[1] Bramson, M. and Griffeath, D. Asymptotics for interacting particle systems on $Z^{d}$. Z. Wahrsch. Verw. Gebiete 53, (1980) 183-196. 
[2] Clifford, P. and Sudbury, A. A model for spacial conflict. Biometrika, 60, (1973), 581-588.

[3] Durrett, R. Lecture notes on particle systems and percolation. Belmont, CA: Wadsworth (1988).

[4] Durrett, R. Probability: Theory and examples. Belmont, CA: Wadsworth (1996).

[5] Harris, T.E. Additive set-valued Markov processes and graphical methods. Ann. Probab., 6, (1978), 355-378.

[6] Harrison, R.G. it Hibrid zones: windows on evolutionary process. In D. Futuyama and J. Antonovics (eds), Oxford Surveys in Evolutionary Biology. Oxford: Oxford University Press (1990) .

[7] Holley, R.A. and Ligett,T.M. Ergodic theorems for weakly interacting systems and the voter model. Ann. Prob., 3, (1975), 643-663.

[8] Liggett, T.M. Interacting Particle Systems. Springer, New York (1985).

[9] L.R.G. Fontes, M. Isopi, M. Isopi, C.M. Newman, K. Ravishankar, The Brownian web: characterization and convergence, Annals og Probability 32, 2857-2883. Springer, New York (2004).

[10] Spitzer, F. Principles of random walk, New York: Spinger-Verlag (1976).

[11] S. Belhaouari, T. Mountfor. Tightness of the interface for one-dimensional voter models.Proceeding of the London Mathematical Society, volume 94, number 2, (2006), pages 421-442

[12] S. Belhaouari, T. Mountfor, R. sun and G. Valle. Convergence results and sharp estimates for the voter model interface. Oxford Journal, Electronic Journal of Probability vol.11, Paper no. 30, (2006), pages 768-801 
